\section{Development of CNS leukaemia in acute myeloid leukaemia in childhood}

It is generally agreed that in acute myeloid leukaemia (AML), as in acute lymphoblastic leukaemia (ALL), the central nervous system (CNS) may be invaded, giving rise to meningeal leukaemia or to localized infiltration. The incidence of such spread has been thought to be low, and because of the generally poor prognosis of AML it has not been considered necessary to give prophylactic treatment to the CNS as is now customary in ALL.

In an attempt to estimate the incidence of CNS involvement in AML in childhood, centres collaborating in trials organized by the Medical Research Council collected data on cases of AML in children diagnosed in the years 1969-1973 inclusive. 130 cases were reported below the age of 14 years, with a fairly even distribution throughout this age range (Table). There were only 7 cases below the age of one year. There was a slight preponderance of males over females.

\section{TABLE}

AML in childhood: MRC 1969-1973

\begin{tabular}{l|r|r|r|r}
\hline & \multicolumn{3}{|c|}{ Age (yr) } & Total \\
\cline { 2 - 4 } & $<3$ & $3-8$ & $9-14$ & \\
\hline No. & 36 & 54 & 40 & 130 \\
With CNS prophylaxis & 1 & 7 & 4 & 12 \\
CNS disease & 6 & 8 & 7 & 21 \\
\hline
\end{tabular}

The cytological types of disease included myeloblastic, myelocytic, and myelomonocytic types, and erythroleukaemia. The diagnosis was in all cases unequivocal as it has been the practice in MRC trials to regard cases of dubious cytological type in patients under 20 years of age as ALL for purposes of treatment. 5 of the patients had originally been submitted for inclusion in a trial of treatment for ALL, but in general the bias of diagnosis and classification is in the opposite direction.

Twelve cases received some sort of prophylactic treatment directed at the CNS and the survival of the remaining 118 is plotted in the Fig. It will be

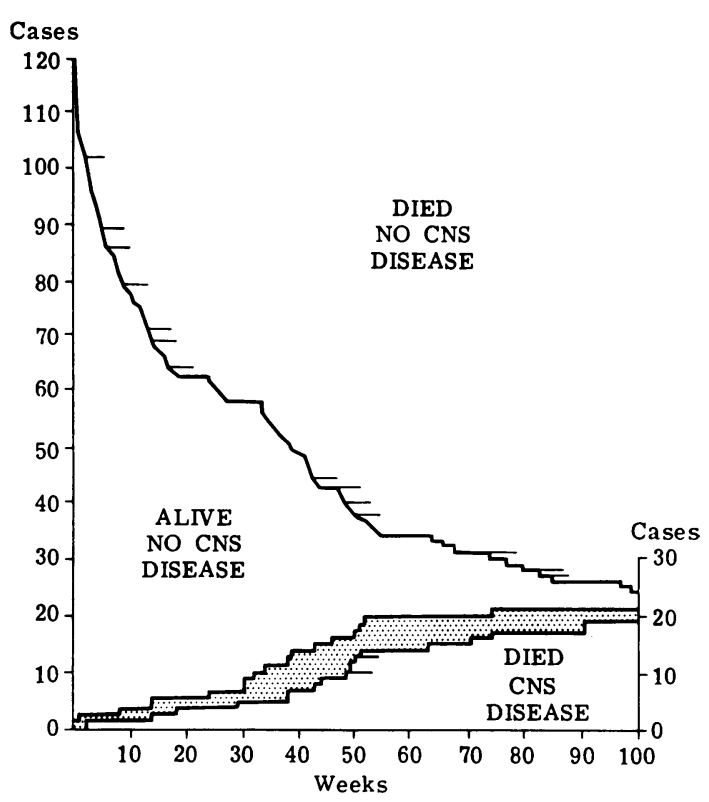

ALIVE CNS DISEASE

Fig.-Survival of 118 children with acute myeloid leukaemia, aged 0-14 years, $(A M L)$ not having had CNS prophylaxis. 22 patients who were still alive at the most recent follow-up are indicated by horizontal bars or have reached 100 weeks.

seen that the cumulative incidence of CNS disease at 2 years is not large-21 cases-but this total has to be related to the pattern of survival. Early mortality and a poor rate of remission produce a median survival of 21 weeks at which time 55 cases have died without CNS involvement, 4 cases have died with CNS involvement, and a further 2 are alive but with diagnosed CNS disease. By one year there are only 23 survivors but the cumulative incidence of CNS disease has now reached 20, including 6 of the survivors. (Note that all these figures will improve slightly as those still alive (indicated by horizontal bars) survive beyond the latest follow-up.

The form of disease was essentially similar to that seen in ALL with headaches, vomiting, and papilloedema as common signs and with leukaemic cells in the cerebrospinal fluid. Nerve palsies and other localizing signs were also reported. In 2 
cases CNS involvement was diagnosed in the terminal stages on clinical evidence aloneheadaches, vomiting, and papilloedema in one case, blindness in another; in 2 further cases undiagnosed involvement of the CNS was found at necropsy (at weeks 38 and 74).

Throughout this period the form of treatment was varied, the commonest regimens being combinations such as those described by Gee, $\mathrm{Yu}$, and Clarkson (1969), Crowther, et al. (1970), the Medical Research Council (1974) and others. As far as can be determined no particular regimen was associated with a particularly high or low incidence of CNS disease. However, among the 12 children who received some sort of CNS prophylaxis there have so far been no cases of meningeal leukaemia. Prophylaxis has included cranial (and spinal) irradiation and intrathecal methotrexate and/or cytosine arabinoside. Taking the period starting 6 weeks after diagnosis, which was the usual point at which CNS prophylaxis began, a comparison can be made of the relative incidence of CNS leukaemia in the time at risk between those who did and those who did not have CNS prophylaxis. Among 82 cases without prophylaxis, totalling 2707 weeks at risk, there were 15 cases of CNS disease, an incidence of 1 case per 180 weeks: this can be compared with 12 cases who received some sort of prophylactic treatment to the CNS, none of whom developed CNS leukaemia during a total of 544 weeks at risk.

\section{Summary}

In 130 cases of acute myeloid leukaemia in children below the age of 14 years in Great Britain, there were 21 cases in which the central nervous system was involved. The incidence and timing is similar to that of acute lymphoblastic leukaemia; in a small number of patients who received prophylactic treatment, involvement of the central nervous system was prevented.

REFERENCES

Crowther, D., Bateman, C. J. T., Vartan, C. P., Whitehouse, J. M. A., Malpas, J. S., Fairley, G. H., and Scott, R. B. (1970). Combination chemotherapy using $\mathrm{L}$-asparaginase, daunorubicin and cytosine arabinoside in adults with acute myelogenous leukaemia. British Medical fournal, 4, 513.

Gee, T. S., Yu, K-P., and Clarkson, B. D. (1969). Treatment of adult acute leukaemia with arabinosylcytosine and thioguanine Cancer, 23, 1019.

Report of the Medical Research Council's Working Party on Leukaemia in Adults (1974). Treatment of acute myeloid leukaemia with daunorubicin, cytosine arabinoside, mercaptopurine, I-asparaginase, prednisone and thioguanine: results of treatment with five multiple-drug schedules. British fournal of Haematology, 27, 373.
H. E. M. KAY ${ }^{\star}$ on behalf of the Medical Research Council's Working Partyt on Leukaemia in Childhood

*Correspondence to H.E.M.K., Royal Marsden Hospital, Fulham Rd., London SW3 6JJ.

tDr. K. D. Bagshawe, Dr. J. M. Bridges, Professor Neville Butler, Dr. J. M. Chessells, Dr. P. F. Deasy, Sir Richard Doll, Dr. P. Emerson, Dr. H. W. Everley Jones, Dr. D. I. K. Evans, Dr. D. M. T. Gairdner, Dr. D. A. G. Galton, Dr. R. J. Guyer, Professor R. M. Hardisty (Secretary), Dr. C. B. Howarth, Professor J. M. Hutchison (Chairman), Dr. E. M. Innes, Dr. P. Morris Jones, Dr. T. J. McElwain, Dr. I. C. M. MacLennan, Dr. J. Martin, Professor I. C. S. Nomand, Dr. P. G. Smith, Dr. J. Stuart, Dr. E. N. Thompson, Dr. M. L. N. Willoughby.

\section{Multiple major cerebral artery thromboses with profound thrombocytopenia in acute leukaemia}

Cytotoxic, corticosteroid, and antibiotic therapy in leukaemia are associated with an increase in fungal infections (Bodey, 1966). Having to deal with the clinical problems of thrombocytopenia and bleeding in leukaemia is not uncommon. In this situation it is very unusual to find at necropsy extensive antemortem thrombosis in the cerebral arterial circulation.

\section{Case report}

A $5 \frac{1}{2}$-year-old girl was found to have acute lymphatic leukaemia (ALL), with a haemoglobin of $7 \cdot 4 \mathrm{~g} / \mathrm{dl}$, a platelet count of $30000 / \mathrm{mm}^{3}$, and leucocytes at $4900 /$ $\mathrm{mm}^{3}$ with $2 \%$ neutrophils, $68 \%$ lymphocytes, and $30 \%$ blast cells. Bone marrow biopsy showed that $96 \%$ of the nucleated cells were blasts.

She was treated over a 9-week period, according to the protocol of the MRC UKALL III Ordinary Schedule with intravenous vincristine and oral prednisone to induce remission, followed by oral 6-mercaptopurine, intravenous asparaginase, intrathecal methotrexate, and cerebral irradiation. After 4 weeks her peripheral blood and bone marrow had returned to normal During the 10th week she had one dose of oral methotrexate after which her leucocyte and platelet counts began to fall abruptly.

She was readmitted during the 12th week with fever, respiratory distress, widespread purpura, and rectal bleeding. She was anaemic ( $\mathrm{Hb} 5 \mathrm{~g} / \mathrm{dl}$ ), leucopenic (total white blood count $2000 / \mathrm{mm}^{3}$ ) and thrombocytopenic (platelets $20000 / \mathrm{mm}^{3}$ ). A blood culture grew meningococci. The bone marrow was hypoplastic. All cytotoxic therapy was stopped and she was given penicillin, sulphadimidine, and prednisone. While in this thrombocytopenic state she fell out of bed and developed a large frontal haematoma, the blood later gravitating down to surround both eyes. The skin overlying the 